\title{
Application of the Isobaric Mass Multiplet Equation to the rp process in Nuclear Astrophysics
}

\author{
Werner Richter* \\ iThemba LABS,P.O.Box 722, Somerset West 7129, South Africa \\ University of the Western Cape, Private Bag X17, Bellville 7535, South Africa \\ E-mail: richteresun.ac.za

\section{B. Alex Brown} \\ Department of Physics and Astronomy, and National Superconducting Cyclotron Laboratory, \\ Michigan State University, East Lansing, Michigan 48824-1321, USA \\ E-mail: brownenscl.msu.edu
}

In many cases levels of proton-rich nuclei participating in rp processes have not been measured and one has to rely on theory to estimate the reaction rates. However, the Isobaric Mass Multiplet Equation (IMME) affords a reliable method of obtaining levels in the final $\mathrm{T}=1$ nucleus of a (p, $\gamma$ ) reaction in terms of experimental energies of the isobaric analog partners and a small coefficient $\mathrm{c}$ (typically 150 - $300 \mathrm{keV}$ ) that can be calculated. The power of the IMME method, which is mainly empirically based with a small theoretical component, to estimate energies of nuclei participating in the rp process is emphasized by way of illustrating favorable cases where the method works particularly well. We demonstrate the usefulness of the application of the IMME to three rp reactions ${ }^{25} \mathrm{Al}(\mathrm{p}, \gamma){ }^{26} \mathrm{Si},{ }^{35} \mathrm{Ar}(\mathrm{p}, \gamma){ }^{36} \mathrm{~K}$ and ${ }^{29} \mathrm{P}(\mathrm{p}, \gamma){ }^{30} \mathrm{~S}$. Because of the semi-empirical nature of the method, it should be stressed that its application depends on knowing energies of the analog states of the nucleus in question. Alternatively, one has to resort to shell-model calculations with reliable two-body interactions.

51st International Winter Meeting on Nuclear Physics

21-25 January 2013

Bormio (Italy)

\footnotetext{
${ }^{*}$ Speaker.
} 


\section{Introduction}

A process such as the $\mathrm{rp}(\mathrm{p}, \gamma)$ reaction is generally dominated by resonant capture to excited states above the particle-emission threshold and therefore depends critically on the nuclear properties of the levels involved. The Isobaric Mass Multiplet Equation (IMME) affords a reliable method of obtaining levels in the $\left(T_{z}=-1\right)$ nuclei for the $(\mathrm{p}, \gamma)$ reactions in terms of the isobaric analog partners and a relatively small (typically $150-300 \mathrm{keV}$ ) IMME coefficient $c$ that can be calculated [1]. The $(\mathrm{p}, \gamma)$ rate depends on the proton-decay and gamma-decay widths that are often not measured experimentally. Values obtained from the nuclear shell model can be used if the experimental levels can be matched with their theoretical counterparts. In this paper we use the sd-shell model space with the charge-independent interactions USDA and USDB [2] supplemented by Coulomb and charge-dependent interactions obtained in Ref. [3].

According to the IMME

$$
B=a+b T_{z}+c T_{z}^{2},
$$

where $B$ is the binding energy of a state. For the three $\mathrm{T}=1$ isobaric states one can then, with $T_{z}=(N-Z) / 2$, substitute $T_{z}=1,0,-1$ alternately, and by rearranging

$$
B_{p}=2 B_{o}-B_{n}+2 c
$$

for the proton-rich member, where $c$ can be expressed as

$$
c=\left(B_{n}+B_{p}-2 B_{o}\right) / 2 \text {. }
$$

As a specific example, for ${ }^{26} \mathrm{Si}$ one has

$$
B_{t h}\left({ }^{26} \mathrm{Si}\right)=2 B\left({ }^{26} \mathrm{Al}\right)-B\left({ }^{26} \mathrm{Mg}\right)+2 c_{t h} .
$$

\section{The two-body interactions employed}

In the paper of Ormand and Brown [3] they start with a known isoscalar Hamiltonian, e.g. USD, and calculate the one-body transition densities (OBTD) and two-body transition densities (TBTD) for a set of nuclei in a particular model space, e.g. the sd shell for USD. Next a semiempirical isospin non-conserving interaction (INC) with standards terms is composed and treated as a perturbation. The various strengths of the terms are obtained from least-squares fits to data (the data being coefficients of the Isobaric Mass Multiplet Equation (IMME)). The $T=1$ part of the two-body $\mathrm{NN}$ interaction is written as a sum of isoscalar, isovector and isotensor parts with each term a product of an isospin part and an interaction $\mathrm{v}^{k}$. Each $\mathrm{v}^{k}$ is expanded as a sum of products of a strength $\mathrm{S}_{\mu}$ and an interaction $\mathrm{V}_{\mu}(r)$ (Eq. 3.1 in [3]). The interaction terms postulated are $\mathrm{V}_{0}$, the isoscalar part of the initial isoscalar two-body Hamiltonian which is known, the Coulomb potential $\mathrm{V}_{C}$, and $\mathrm{V}_{\pi}$ and $\mathrm{V}_{\rho}$ meson exchange terms with Yukawa forms. The tensor components of the interaction are then rewritten in the proton-neutron formalism and expressed i.t.o. the interactions between protons and neutrons. One obtains a charge-asymmetric part of the NN interaction $\mathrm{v}^{(1)}$ $=\left(\mathrm{v}^{(p p)}-\mathrm{v}^{(n n)}\right)$ and a charge-dependent part $\mathrm{v}^{(2)}=\left(\mathrm{v}^{(p p)}+\mathrm{v}^{(n n)}-2 \mathrm{v}^{(p n)}\right)$ which break isospin symmetry. 


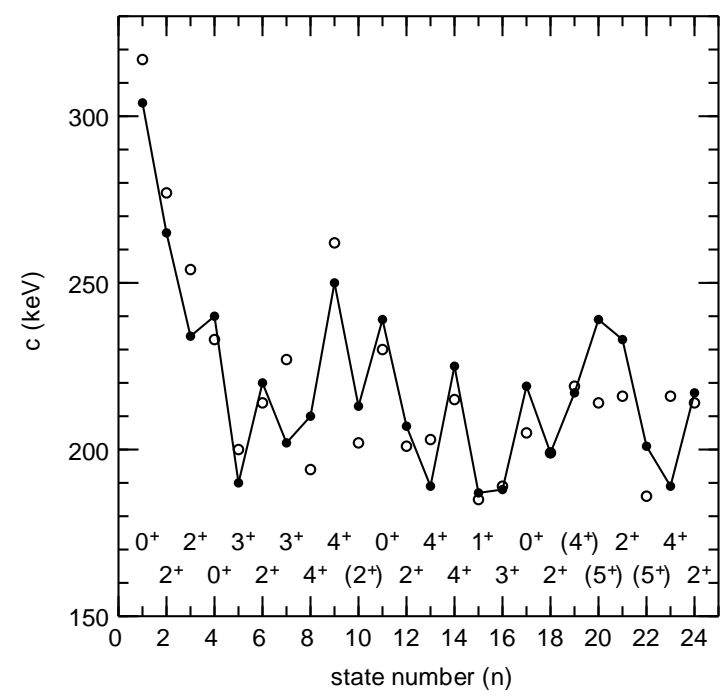

Figure 1: $c$-coefficients from the isobaric mass multiplet equation (IMME: $E=a+b T_{z}+c T_{z}^{2}$ ) versus state number (in order of increasing energy) in ${ }^{26} \mathrm{Si}$ based on experimental energies (closed circles) and energies calculated from USDB (open circles).

The $\mathrm{b}$ and $\mathrm{c}$ coefficients of the Isobaric Mass Multiplet Equation (IMME) can be written in terms of the isovector single-particle energies $\varepsilon^{(1)}=\varepsilon^{(p)}-\varepsilon^{(n)}$ and the strength parameters $\mathrm{S}_{\mu}$. Their values are then determined from a least-square fit between the theoretical and empirical $b$ and c coefficients in a particular model space. For the nuclei considered in [3], $A=18-22$ and $A=34-39$, the $42 b$-coefficients were reproduced with an rms deviation of $27 \mathrm{keV}$ and the $26 c$-coefficients were reproduced with an rms deviation of $9 \mathrm{keV}$. There is considerable state-dependence in the $c$-coefficients (ranging in values from $130 \mathrm{keV}$ to $350 \mathrm{keV}$ ) that is nicely reproduced by the calculations (see Fig. 9 in [3]).

The INC Hamiltonian is then added to the isoscalar Hamiltonian, originally USD in the sd shell. In our case we use USDA and USDB for the charge-independent parts of the two-body interaction. These composite interactions are called USDA-cdpn and USDB-cdpn in NuShellX 邨, implying that they are charge-dependent and that calculations are done in the pn formalism.

\section{3. ${ }^{26} \mathrm{Si}$ as the final nucleus}

Because many levels in ${ }^{26} \mathrm{Si}$ have uncertainties in terms of energy, spin and parity, a procedure often adopted is to make assignments in ${ }^{26} \mathrm{Si}$ based on known levels in the mirror nucleus ${ }^{26} \mathrm{Mg}$. We have also made use of experimental information on the levels of excited states in ${ }^{26} \mathrm{Si}$ from Ref. [5]. Using the new $s d$-shell interactions USDA and USDB [2], as well as the older USD interaction [6], assignments between theory and experiment of corresponding levels in ${ }^{26} \mathrm{Mg}$ levels have been confirmed, and new ones suggested [ []. It has also been shown previously that the new interactions reproduce most observables in the sd shell reliably, and in some cases better than USD [8].

In Fig. (1) values of $c$ from experiment and theory are compared for states in ${ }^{26} \mathrm{Si}$ ordered according to increasing experimental energy. The experimental values are obtained for states where 


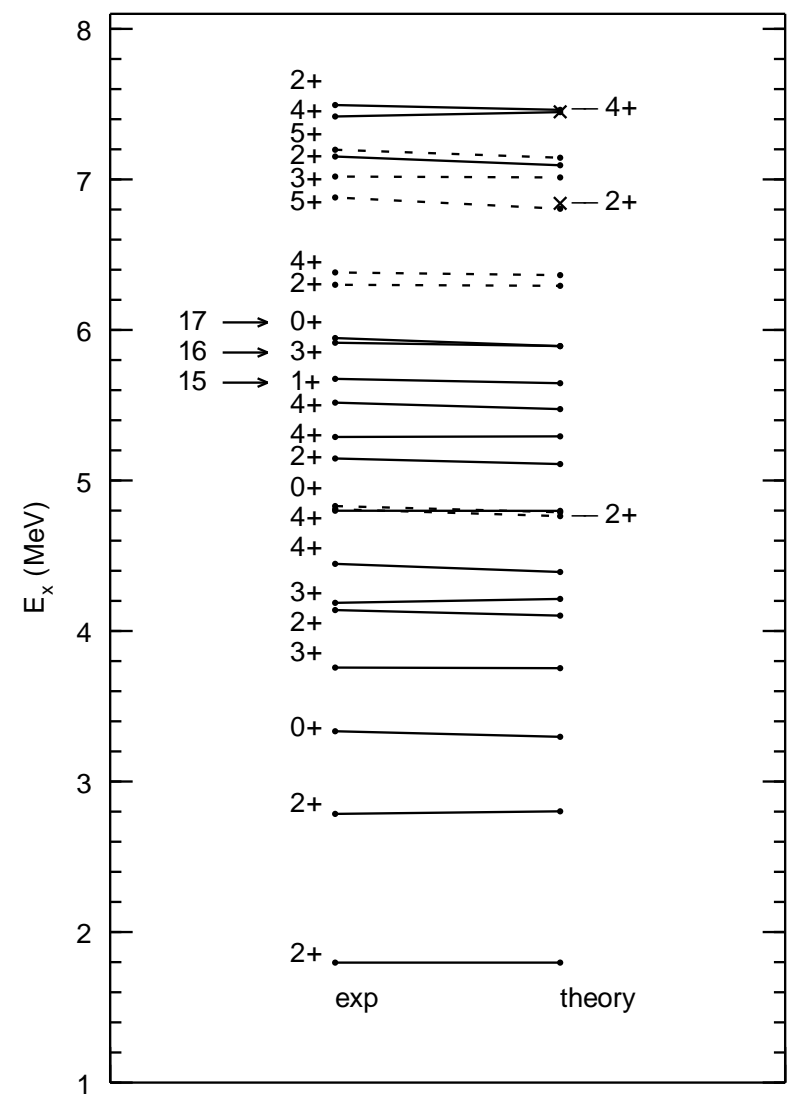

Figure 2: Adopted experimental excitation energies in ${ }^{26} \mathrm{Si}$ [5] versus predicted energies $E_{t h}$ based on experimental binding energies of ${ }^{26} \mathrm{Mg}$ and ${ }^{26} \mathrm{Al}$ and the theoretical $c$-coefficient (from USDB-cdpn) (Eq.

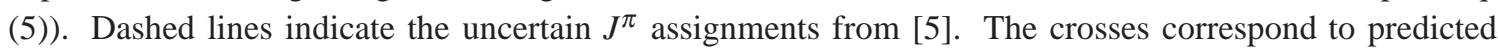
energies without experimental counterparts.

all three members of the multiplet are known. In general a good correspondence can be seen, the largest deviations being less than $30 \mathrm{keV}$. There is considerable state dependence with $c$ values ranging from $300 \mathrm{keV}$ (for the $0^{+}$ground state) down to $180 \mathrm{keV}$. This IMME method was used in [9] for the $\mathrm{T}=1$ states of the odd-odd nuclei with mass 28,32 and 36 . The agreement with experiment [Fig. (1)] for our even-even case appears to be better than obtained in [9] for the oddodd cases.

Fig. (2) shows the excitation energies for ${ }^{26} \mathrm{Si}$ obtained from Eq. (2) on the right compared to experiment on the left. The calculated values can then be used as a guide to the correct spin/parity assignments for measured levels in ${ }^{26} \mathrm{Si}$. Where no levels in ${ }^{26} \mathrm{Si}$ are known, levels can be predicted. Two such levels are indicated by crosses in Fig. (2). The three levels that are just above the protondecay separation energy of $5.51 \mathrm{MeV}$ and of potential importance for the capture reaction at low temperatures are indicated by the arrows in Fig. (2).

The well-established experimental energies and energies based on the IMME are used as input for the (r,p) rate calculations. The results for ${ }^{26} \mathrm{Si}$ have been given in Ref. [1]]. 


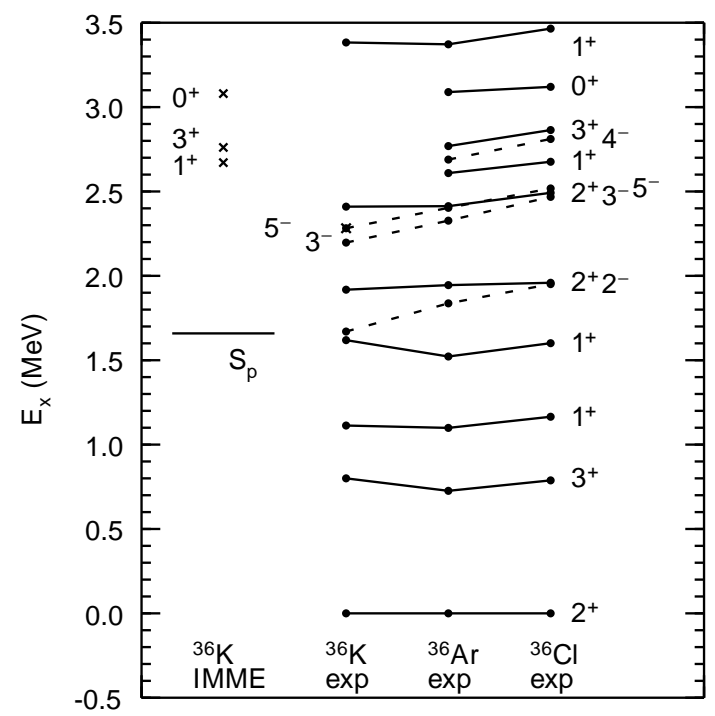

Figure 3: Experimental energies of the isobaric $\mathrm{T}=1$ triplets for $\mathrm{A}=36$. The energies of ${ }^{36} \mathrm{Ar}$ are relative to the lowest $2^{+} \mathrm{T}=1$ state at $6.611 \mathrm{MeV}$. Negative parity states are connected by dashed lines. The solid lines connect positive parity states considered to be analogs on the basis of our IMME predictions. The proton separation energy in ${ }^{36} \mathrm{~K}$ is shown by the horizontal line on the left-hand side. The data are from Endt [11] except for those above the proton separation energy in ${ }^{36} \mathrm{~K}$ for which we use the newer values from Wrede et al. [10]. The cross on the $2.282 \mathrm{MeV} 5^{-}$state in ${ }^{36} \mathrm{~K}$ indicates that this level was associated with the $2_{3}^{+}$state by Wrede et al. We associate the $2_{3}^{+}$level with the higher state at $2.446 \mathrm{MeV}$. The levels labeled

${ }^{36} \mathrm{~K}$ IMME are based on Eq. 2 with experimental binding energies of ${ }^{36} \mathrm{Cl}$ and ${ }^{36} \mathrm{Ar}$ and with the theoretical $c$-coefficient (Eq. (3)). The crosses correspond to predicted energies without experimental counterparts.

\section{4. ${ }^{36} \mathrm{~K}$ as the final nucleus}

Fig. (3) shows the experimental excitation energies of the $T=1$ analog states for $A=36$. A number of levels of ${ }^{36} \mathrm{~K}$ measured recently by Wrede et al [10] above the proton separation are included, and all other excitation energies are from Ref. [11]. The cross on the $2.282 \mathrm{MeV}^{-}$ state in ${ }^{36} \mathrm{~K}$ indicates that this level was associated with the $2_{3}^{+}$state by Wrede et al. Our reasons for associating the $2_{3}^{+}$level with the higher state at $2.446 \mathrm{MeV}$ state are discussed in detail in Ref. [12], but are based on comparison with the IMME. The levels labeled ${ }^{36} \mathrm{~K}$ IMME are based on Eq. (2) with the experimental binding energies of ${ }^{36} \mathrm{Cl}$ and ${ }^{36} \mathrm{Ar}$ and the theoretical $c$-coefficient (Eq.

(3)). The crosses correspond to predicted energies without experimental counterparts.

In the present case there are two negative parity states, $3^{-}$and $5^{-}$, as shown in Fig. (3), close to some of the important resonances, and their contributions should be taken into account. In view of the correspondence between mirror states for $\mathrm{A}=36$ it would be reasonable to substitute experimental values of the spectroscopic factors and lifetimes from the mirror nucleus ${ }^{36} \mathrm{Cl}$ in cases where a calculation is not feasible. In this way the contributions from these negative parity levels can be taken into account approximately.

In Fig. (4) the reaction rates leading to ${ }^{36} \mathrm{~K}$ are shown. It should be noted that the contribution 


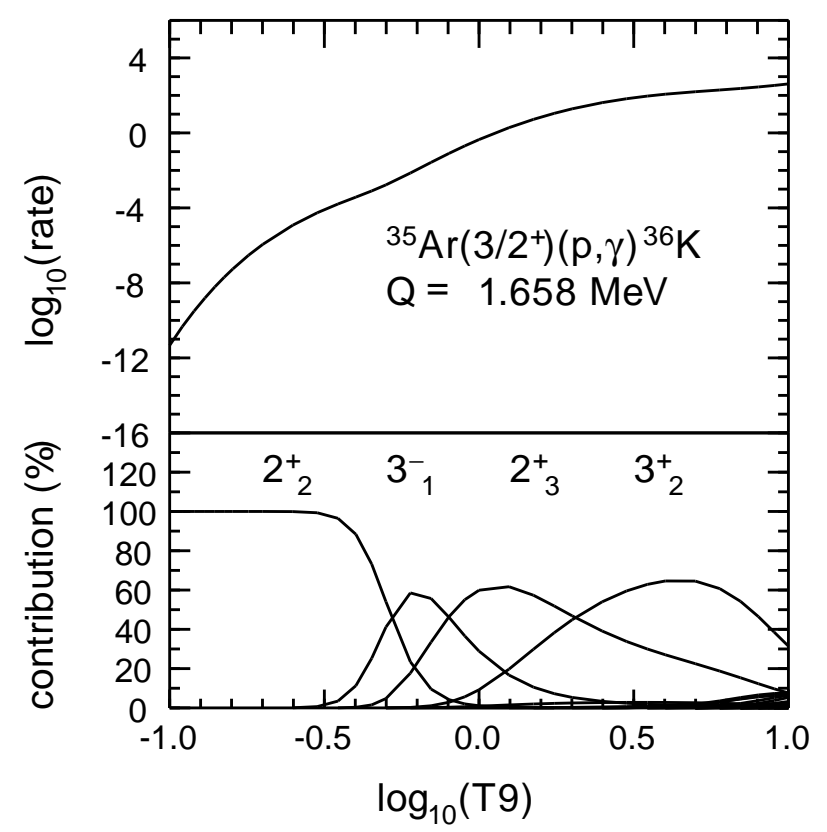

Figure 4: The total rp reaction rate versus temperature T9 (GigaK) (top panel) and the contribution of each of the final states (lower panel) with usdb-cdpn. $\Gamma_{\gamma}$ was calculated for ${ }^{36} \mathrm{~K}$ levels.

of the negative parity state $3^{-}$is significant and cannot be neglected, even if it has to be based on measured spectroscopic factors and gamma widths of the mirror nucleus ${ }^{36} \mathrm{Cl}$.

\section{5. ${ }^{30} \mathrm{~S}$ as the final nucleus}

Our normal procedure is to use Eq. (2) to predict energies and confirm spin assignments in ${ }^{30} \mathrm{~S}$. However, based on the recent data for ${ }^{30} \mathrm{~S}$ we found several inconsistencies when using the proposed experimental $T=1$ assignments for levels above five $\mathrm{MeV}$ in ${ }^{30} \mathrm{P}$. Thus, for $A=30$ we start with an investigation of the $\left(J^{\pi}, T\right)$ assignments for levels in ${ }^{30} \mathrm{P}$.

In Table I a summary is given of the $T=1$ triplets for $A=30$. Experimental energies for ${ }^{30} \mathrm{Si}$ and ${ }^{30} \mathrm{P}$ are are taken from the Nuclear Data Sheets (NDS) [14] unless otherwise indicated. The levels are numbered by $n$ according to their well-established ordering in ${ }^{30} \mathrm{Si}$. The levels for a given $J^{\pi}$ value are numbered by $k$. The ${ }^{30} \mathrm{P}$ energies and $J^{\pi}$ for the states below $5 \mathrm{MeV}$, as well as the $3^{+}(n=8, k=2)$ level at $5219 \mathrm{keV}$ are taken from Lotay et al. [15]. The energies and $J^{\pi}$ values for other states above $5 \mathrm{MeV}$ are taken from Almaraz-Calderon et al. [16]. Above $6 \mathrm{MeV}$ there are many states in ${ }^{30} \mathrm{~S}$ whose $J^{\pi}$ values are uncertain. The states between 6 and $7 \mathrm{MeV}$ given in Table I are those expected from the well-known levels in ${ }^{30} \mathrm{Si}$.

Having made several reassignments for levels in ${ }^{30} \mathrm{P}$ [17], the $c$ coefficients for 13 positiveparity levels in Table I are shown in Fig. 5. There is good agreement between experiment and theory except for the $0^{+} n=10$ state. The energy of this state in ${ }^{30} \mathrm{P}$ needs to be confirmed experimentally. As in Ref. [3] there is significant state dependence with $c$ values from experiment ranging from 


\begin{tabular}{|c|c|c|c||c|c|c||c||}
\hline$n$ & $J^{\pi}$ & $k$ & ${ }^{30} \mathrm{P}$ & ${ }^{30} \mathrm{Si}$ & ${ }^{30} \mathrm{P}-677 \mathrm{keV}$ & ${ }^{30} \mathrm{~S}$ & ${ }^{30} \mathrm{~S}$ \\
\hline & & & $\exp$ & $\exp$ & $\exp$ & $\exp$ & USDB-cdpn \\
\hline 1 & $0^{+}$ & 1 & 677 & 0 & 0 & 0 & 0 \\
2 & $2^{+}$ & 1 & 2937 & 2235 & 2260 & 2210 & 2244 \\
3 & $2^{+}$ & 2 & 4182 & 3498 & 3505 & 3404 & 3485 \\
4 & $1^{+}$ & 1 & 4502 & 3769 & 3825 & 3677 & 3976 \\
5 & $0^{+}$ & 2 & 4468 & 3788 & 3791 & 3668 & 3871 \\
6 & $2^{+}$ & 3 & 5576 & 4810 & 4899 & 4809 & 4805 \\
7 & $3^{+}$ & 1 & $5509(2,3)$ & 4830 & 4832 & 4688 & 4825 \\
8 & $3^{+}$ & 2 & $6006\left(3^{+}\right)$ & 5231 & $5329\left(3^{+}\right)$ & 5219 & 5111 \\
9 & $4^{+}$ & 1 & $5934\left(3^{+}\right)$ & 5279 & $5257\left(3^{+}\right)$ & 5132 & 5278 \\
10 & $0^{+}$ & 3 & $6050(10)(\mathrm{a})$ & 5372 & {$[5373(10)](\mathrm{a})$} & 5218 & 5487 \\
11 & $3^{-}$ & 1 & 6093 & 5487 & 5414 & 5312 & \\
12 & $2^{+}$ & 4 & $6268(2-)$ & 5614 & 5593 & 5382 & 5867 \\
13 & $4^{+}$ & 2 & 6597 & 5951 & 5921 & 5836 & 5860 \\
14 & $4^{-}$ & 1 & 7049 & 6503 & 6372 & $(6225) *$ & \\
15 & $2^{+}$ & 5 & & 6537 & & & 6497 \\
16 & $2^{-}$ & 1 & $7223\left(2^{-}\right)$ & 6641 & $6546\left(2^{-}\right)$ & $(6435) *$ & \\
17 & $0^{+}$ & 4 & $7207\left(0^{+}\right)$ & 6642 & 6530 & 6326 & 6725 \\
18 & $1^{-}$ & 1 & $7178\left(1^{-}\right)$ & 6744 & $6501\left(1^{-}\right)$ & $(6242) *$ & \\
19 & $3^{+}$ & 3 & & 6865 & & & 6940 \\
20 & $2^{+}$ & 6 & & $6915\left(2^{+}\right)$ & & & 7024 \\
21 & $5^{+}$ & 1 & & 6999 & & & 6996 \\
\hline
\end{tabular}

Table 1: Energy levels of the $T=1$ isobaric analog states in $A=30$, and experimental and theoretical c coefficients of the IMME in keV. Excitation energies are given in keV. Error margins are given only when they exceed a few keV. The multiplicity of the states $k$ is determined by USDB-cdpn, and the state number $n$ is in order of increasing energy for ${ }^{30} \mathrm{Si}$, where possible. Ref. [13] is indicated by (a). The other references for the experimental data are discussed in the text. The negative-parity levels indicated by $*$ have energies in ${ }^{30} \mathrm{~S}$ estimated from IMME systematics.

about $170 \mathrm{keV}$ to $276 \mathrm{keV}$. Results for both USDA-cdpn and USDB-cdpn shown in Fig. 5 give some indication of the theoretical uncertainties.

Fig. (6) shows the results for the resonance-capture rate obtained using USDB-cdpn. The three dominant resonances are $3^{+}(1), 2^{+}(3)$ and $2^{+}(4)$. The importance of the $3^{+}(1)$ and $2^{+}(3)$ states was noted in Ref. [18].

\section{Conclusions}

We have demonstrated the application of the IMME method to three $\mathrm{rp}(\mathrm{p}, \gamma)$ reactions. In the case of ${ }^{26} \mathrm{Si}$ as the final nucleus, we have correlated levels predicted by the IMME with measured levels with uncertain spin/parity assignments in order to make more definite assigments. For ${ }^{36} \mathrm{~K}$ most energy levels in the resonance region have been measured, but comparison with the IMME 


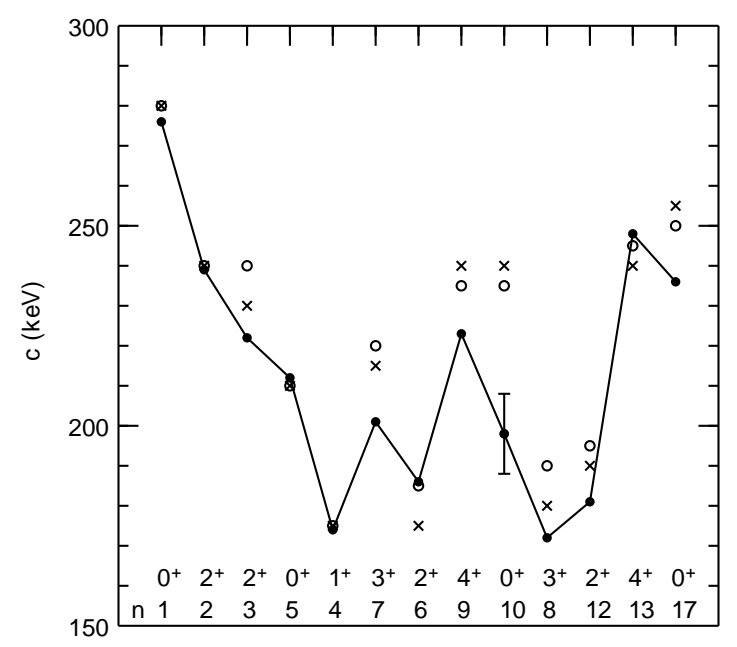

Figure 5: $c$-coefficients from the isobaric mass multiplet equation (IMME: $E=a+b T_{z}+c T_{z}^{2}$ ) for states in ${ }^{30} \mathrm{~S}$ (in order of increasing experimental energy, as in Table I). The coefficients are experimental (closed circles) and theoretical, calculated from USDB-cdpn (open circles) and USDA-cdpn (crosses).

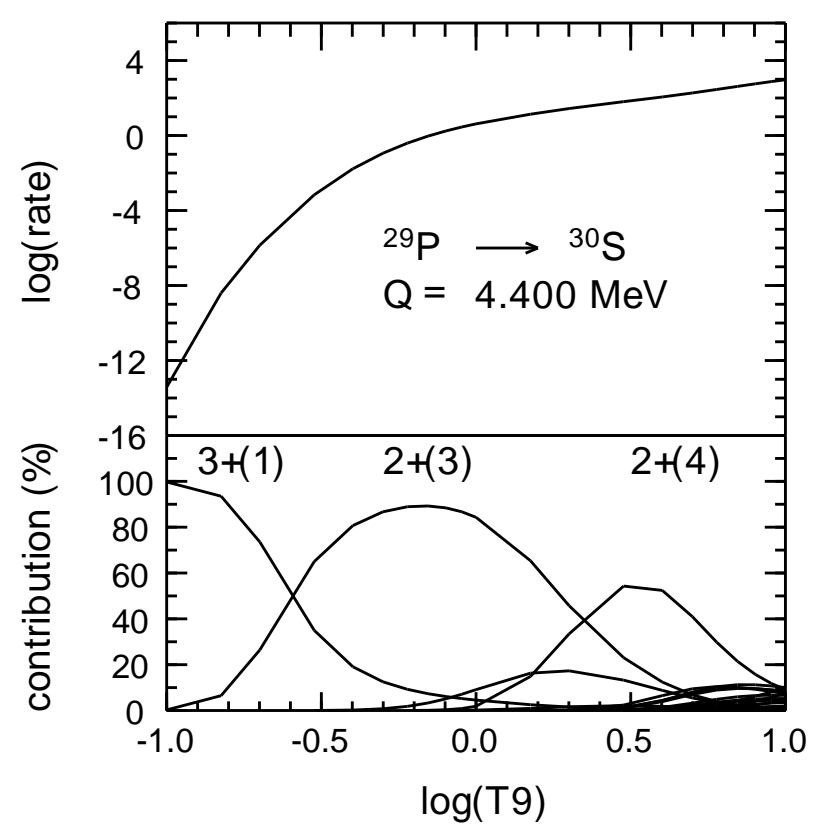

Figure 6: The total rp reaction rate versus temperature T9 (GigaK) (top panel) and the contribution of each of the final states (lower panel) with usdb-cdpn. $\Gamma_{\gamma}$ was calculated for ${ }^{30} \mathrm{~S}$ levels. 
lead us to make some reassignments for some important levels. We also emphasize, in this case, the importance of taking into account the contribution of negative parity states approximately by using lifetimes and spectroscopic factors of the mirror nucleus. In the case of ${ }^{30} \mathrm{~S}$ attempts to apply the IMME indicated many inconsistent assignments for the intermediate $\mathrm{T}=1$ nucleus ${ }^{30} \mathrm{P}$. New assignments for ${ }^{30} \mathrm{P}$ were checked against the IMME, using recent energy values for ${ }^{30} \mathrm{~S}$, and a good correspondence was achieved. The rp reaction rates were calculated for all three reactions using the energies obtained from the IMME as inputs, in addition to the established experimental values.

Acknowledgments This work is partly supported by NSF Grant PHY-1068217, the Joint Institute for Nuclear Astrophysics NSF Grant PHY08-22648, and the National Research Foundation of South Africa Grant No. 76898.

\section{References}

[1] W. A. Richter, B. A. Brown, A. Signoracci and M. Wiescher, Phys. Rev. C 83, 065803 (2011).

[2] B. A. Brown and W. A. Richter, Phys. Rev. C 74, 034315 (2006).

[3] W. E. Ormand and B. A. Brown, Nucl. Phys. A 491, 1 (1989).

[4] www.nscl.msu.edu/ brown/resources/resources.html

[5] A. Matic et al., Phys. Rev. C 82, 025807 (2010).

[6] B. H. Wildenthal, Prog. Part. Nucl. Phys. 11, 5 (1984).

[7] W. A. Richter and B. A. Brown, Phys. Rev. C 80, 034301 (2009).

[8] W. A. Richter, S. Mkhize and B. A. Brown, Phys. Rev. C 78, 064302-1 (2008).

[9] C. Iliadis, P. M. Endt, N. Prantzos and W. J. Thompson, The Astrophysical Jour. 524, 434 (1999).

[10] C. Wrede, J. A. Clark, C.M. Deibel, T. Faestermann, R. Hartenberger, A. Parikh, H.-F. Wirth, S. Bishop, A. A. Chen, K. Eppinger, B. M. Freeman, R. Krucken, O. Lepyoshkina, G. Rugel and K. Setoodehnia, Phys. Rev. C 82, 035805 (2010)

[11] P. M. Endt, Nucl. Phys. A 633, 1 (1998)

[12] W. A Richter and B. A. Brown, Phys. Rev. C 85, 045806 (2012).

[13] B. Ramstein, L. H. Rosier and R. J. De Meijer, Nucl. Phys. A 363, 110 (1981).

[14] http://www.nndc.bnl.gov/ (2012).

[15] G. Lotay, J. P. Wallace, P. J. Woods, D. Seweryniak, M. P. Carpenter, C. J. Chiara, D. T. Doherty, R. V. F. Janssens, T. Lauritsen, A. M. Rogers, and S. Zhu, Phys. Rev. C 86, 042801(R) (2012).

[16] S. Almaraz-Calderon, W. P. Tan, A. Aprahamian, M. Beard, G. P. A. Berg, B. Bucher, M. Couder, J. Görres, S. O’Brien, D. Patel, A. Roberts, K. Sault, and M. Wiescher, Phys Rev. C 86, 065805 (2012).

[17] Private communication, W A Richter, B A Brown.

[18] C. Iliadis, J. M. D’Auria, S. Starrfield, W. J. Thompson, and M. Wiescher, Astrophys. J. Suppl. Ser. 134,151 (2001). 